\title{
Fracture of the tibia after a fibula graft for mandibular reconstruction: a rare complication, report of a case
}

\author{
Ru-shan Goey ${ }^{1}$, Bert van Drunen ${ }^{1}$, Enrike van der Linden ${ }^{1}$, and J. P. Richard van \\ Merkesteyn ${ }^{1}$ \\ ${ }^{1}$ Leiden Universitair Medisch Centrum
}

November 25, 2020

\begin{abstract}
An uncommon complication after a fibula graft for a mandibular reconstruction is a fracture of the tibia. This report describes a case and the treatment of a fracture of the tibia after a partial mandibulectomy and neck dissection, followed by a mandibular reconstruction with a vascularized left fibula graft.
\end{abstract}

\section{Introduction}

The use of a fibula graft for mandibular reconstruction has been described since the 1990s as one of the better alternatives and is one of the most frequently used free flaps in head and neck reconstructions. ${ }^{1,2}$ The free flaps have been described as having a success rate up to more than $95 \%$. The fibula graft has several advantages over other reconstructions like the scapula, radius and iliac crest. It can measure up to a length of bone (up to $25 \mathrm{~cm}$ which is sufficient for a whole mandible) and has a consistent shape with the possibility of a double barrel, leading to good vertical dimensions of the reconstruction. Adequate vascularity and skin can also be obtained for the reconstruction. Another advantage is that the transplant can be harvested simultaneously with a second team as there is enough distance from the team operating on the head-neck. Besides these advantages, donor site morbidity can be described as relatively low by several authors. ${ }^{1-3}$

However in the literature, donor site morbidity of a free fibula flap ranges from 2-57 percent. ${ }^{3-6}$ The morbidity can be divided into early and late complications. Early complications are wound infection, dehiscence or necrosis, compartment syndrome, delayed wound healing, partial or total skin graft loss and abscesses. Late complications are chronic pain, considerable gait abnormality, ankle instability, sensory deficit, leg weakness and limited ankle mobility. Other morbidities could be cold intolerance and poor aesthetics. However, only a small percentage of these complications (about $3 \%$ ) need surgical intervention and most patients have hardly any restrictions in their daily activities. ${ }^{1,3,6,7}$

A less known complication after harvesting a free fibula flap can be a stress fracture of the tibia. Stress fractures are the cause of the inability of the skeletal bone to withstand repetitive loading and are often seen in the lower extremities. They are relatively common among athletes and military recruits and are most frequently seen at the tibia, metatarsals and the fibula. The location of the stress fracture depends on the sort and the level of activity, gender and age. Patients complain about a sudden onset of pain, which is increased by activity and subsides with rest. Besides pain, they usually present with swelling and/or tenderness. ${ }^{8,9}$

The literature describes 14 cases of tibia fractures after a fibula graft excluding this report, which makes it a rare complication. Durst has described and reviewed all cases thus far. ${ }^{10}$ The fractures occurred from 6 weeks till 16 months after the operation. The treatments ranged from conservative therapy by resting, using plaster, splint or a splint to a tibial osteotomy by Durst. ${ }^{10}$ In a systematic review of Ling with a total of 
2534 fibula flaps the tibia fracture wasn't described over a 10 -year period of time. ${ }^{7}$ Durst concluded that an estimation of the incidence would be hard because of the very low numbers. ${ }^{10}$ The very low incidence makes it a not standard complication to consent, though it must be kept in mind with the surgeons if symptoms do occur. The aim of this paper is to give a description of the possible complication of a tibia fracture after harvesting a fibula graft with a case report.

\section{Case report}

A 58 year old man, known with Diabetes Mellitus type II, obesity, a knee prosthesis on the right side, hypertension and renal disfunction, presented with a T4N1M0 squamous cell carcinoma in the left mandible in November 2006. He underwent a partial mandibulectomy and neck dissection on the left side in January 2007, followed by a mandibular reconstruction with a vascularized left fibula graft fixated with a 2.4 AO plate with 4 screws on both sides (Fig 7). Postoperatively the patient developed renal failure for which he was treated with haemodialysis. Afterwards he was treated postoperatively with $66 \mathrm{~Gy}$ of loco-regional radiotherapy.

Eight months later in September 2007 the patient heard a crack in his left leg while elevating out of a sofa and couldn't use his leg anymore. He had already been complaining about pain in the lower left leg for a few weeks. Radiological investigation showed a midshaft fracture of the tibia without osteolysis (Fig 1). A possibility for other pathology like a metastasis was ruled out by x-rays and a skeletal scintigraphy. No medication was found that could relate to a medication related fracture e.g. Prednisone. Thus, he was diagnosed with a tibia stress fracture on the left side. The patient was operated and an $36 \mathrm{~cm}$ unreamed tibia nail diameter 10 was placed (Fig 2).

Figure 1a Anteroposterior and lateral radiography with a tibia fracture 8 months after a free fibula flap preoperatively

Figure 1b Anteroposterior and lateral radiography with a tibia fracture 8 months after a free fibula flap preoperatively

Figure 2a Anteroposterior and lateral radiography following a unreamed tibia nail

Figure 2b Anteroposterior and lateral radiography following a unreamed tibia nail

Three months later in December 2007 the patient heard another crack while walking in the living room and was unable to walk after this. The x-ray showed a fracture of the distal part of the tibia at the end of the nail (Fig 3). The nail was revised to a longer one from 36 to $38 \mathrm{~cm}$ and the patient was treated with a cast (Fig 4).

Figure 3a Anteroposterior and lateral radiography showing a distal fracture of the tibia at the end of the unreamed tibia nail

Figure $3 \mathrm{~b}$ Anteroposterior and lateral radiography showing a distal fracture of the tibia at the end of the unreamed tibia nail

Figure 4a Anteroposterior and lateral radiography after revision of the unreamed tibia nail

Figure 4b Anteroposterior and lateral radiography after revision of the unreamed tibia nail

After 3 months in March 2008 during a check-up it appeared there was a malposition of the distal tibia (Fig 5). The distal screws were removed and the tibia was repositioned and casted again (Fig 6). Follow-up was done till December 2008 until the patient recovered without any further complications and was able to mobilise fully.

Figure 5a Anteroposterior and lateral radiography demonstrating the malposition of the distal tibia

Figure 5b Anteroposterior and lateral radiography demonstrating the malposition of the distal tibia

Figure 6a Anteroposterior and lateral radiography following repositioning and casting of the tibia 
Figure $6 \mathrm{~b}$ Anteroposterior and lateral radiography following repositioning and casting of the tibia

In November 2010 the patient presented again with a second primary tumour, T4aN0M0 squamous cell carcinoma of the left maxillary tuberosity. In March 2011 a partial hemimaxillectomy on the left side, reconstructed with a temporal fascial flap, was performed without any re-irradiation.

A third primary tumour, T1N0M0 on the left lateral side of the tongue, was found in 2016 during the patients check-up. Subsequently, the tumour was resected and a split skin graft was used without re-irradiation. In May 2018 the tumour recurred on his tongue. The patient refrained from further treatment. Follow-up was done till September 2018, after which he passed away in November 2018.

Figure 7b Radiographic follow-up with orthopantomographs showing the mandible after the partial mandibulectomy in respectively 2007, four years later in 2011 after removal of the AO plate and 10 years postoperatively in 2017.

Figure 7b Radiographic follow-up with orthopantomographs showing the mandible after the partial mandibulectomy in respectively 2007, four years later in 2011 after removal of the AO plate and 10 years postoperatively in 2017 .

Figure 7c Radiographic follow-up with orthopantomographs showing the mandible after the partial mandibulectomy in respectively 2007, four years later in 2011 after removal of the AO plate and 10 years postoperatively in 2017 .

\section{Discussion}

A tibia fracture after a fibula harvest is rare complication and has only been described 14 times in the literature. The possibility for a metastasis in this case report had to be ruled, which was done by x-rays and a skeletal scintigraphy. After ruling out the diagnosis of a metastasis or a medication related fracture, a tibia fracture after a fibula harvest can generally considered as a stress fracture. This is regarded as a serious complication. Physicians have to keep this complication in mind if symptoms do occur like pain, swelling or tenderness of the lower leg. It would be advised to warn patients with a very active lifestyle or that are sporty or that are overweight to inform them about the risks and this complication. Although the fibula holds many advantages, these patients might need to be informed about the other possible harvests besides the fibula, so they can make a well-informed decision. Postoperatively patients should slowly increase their activity, as to not putting much stress immediately after the operation. A physiotherapist could also be considered in preventing a tibia stress fracture.

A fracture can occur immediately postoperatively till even after 16 months of the operation. Treatment is usually conservative by resting or using a plaster. In the reported case the patient even had 2 fractures which were treated first by using a unreamed tibia nail and secondly by changing the pen and using a cast. After these treaments the patient didn't have any other complications in the lower leg after a follow-up of about one year, showing that treatment eventually can be successful. Another cause in this case report for the tibia fracture could have been overweight. The patient weighed preoperatively $116 \mathrm{~kg}$, which resulted in a BMI of $31.5 \mathrm{~kg} / \mathrm{m}^{2}$. However postoperatively the patient lost a lot of weight (more than 15\%) due to discomfort during mastication/eating and because of radiotherapy, which brought his BMI down to $28.9 \mathrm{~kg} /$ $\mathrm{m}^{2}$. He was continuously monitored by a dietician, but the overweight might have played a role with the tibia fracture in this case report.

In conclusion a tibia fracture after a fibula harvest is a rare and serious condition, however when treated adequately it has a good outcome.

\section{Author Contribution}

RG wrote, and edited all aspects of the manuscript. RM was a major contributor in writing the manuscript. RM, BD and EL were the attending physicians and operators of this patient and provided critical review of the manuscript. All authors read and approved the final manuscript. 


\section{Conflict of interest statement}

None

\section{Acknowledgement statement}

None

\section{References}

1. Harris BN, Bewley AF. Minimizing free flap donor-site morbidity. Curr Opin Otolaryngol Head Neck Surg. 2016;24(5):447-452.

2. Hidalgo DA. Fibula free flap mandibular reconstruction. Clinics in plastic surgery. 1994;21(1):25-35.

3. Li P, Fang Q, Qi J, Luo R, Sun C. Risk Factors for Early and Late Donor-Site Morbidity After Free Fibula Flap Harvest. Journal of Oral and Maxillofacial Surgery. 2015;73(8):1637-1640.

4. Zimmermann CE, Borner BI, Hasse A, Sieg P. Donor site morbidity after microvascular fibula transfer. Clinical oral investigations.2001;5(4):214-219.

5. Shpitzer T, Neligan P, Boyd B, Gullane P, Gur E, Freeman J. Leg morbidity and function following fibular free flap harvest. Annals of plastic surgery. 1997;38(5):460-464.

6. Momoh AO, Yu P, Skoracki RJ, Liu S, Feng L, Hanasono MM. A prospective cohort study of fibula free flap donor-site morbidity in 157 consecutive patients. Plastic and reconstructive surgery.2011;128(3):714-720.

7. Ling XF, Peng X. What is the price to pay for a free fibula flap? A systematic review of donor-site morbidity following free fibula flap surgery. Plastic and reconstructive surgery. 2012;129(3):657-674.

8. Feldman JJ, Bowman EN, Phillips BB, Weinlein JC. Tibial Stress Fractures in Athletes. The Orthopedic clinics of North America.2016;47(4):733-741.

9. Bennell KL, Brukner PD. Epidemiology and site specificity of stress fractures. Clinics in sports medicine. 1997;16(2):179-196.

10. Durst A, Clibbon J, Davis B. Distal tibial fractures are a poorly recognised complication with fibula free flaps. Annals of the Royal College of Surgeons of England. 2015;97(6):409-413.

\section{List of figure captions}

Figure 1ab Anteroposterior and lateral radiography with a tibia fracture 8 months after a free fibula flap preoperatively

Figure 2ab Anteroposterior and lateral radiography following a unreamed tibia nail

Figure 3 ab Anteroposterior and lateral radiography showing a distal fracture of the tibia at the end of the unreamed tibia nail

Figure 4ab Anteroposterior and lateral radiography after revision of the unreamed tibia nail

Figure 5ab Anteroposterior and lateral radiography demonstrating the malposition of the distal tibia

Figure 6ab Anteroposterior and lateral radiography following repositioning and casting of the tibia

Figure 7abc Radiographic follow-up with orthopantomographs showing the mandible after the partial mandibulectomy in respectively 2007, four years later in 2011 after removal of the AO plate and 10 years postoperatively in 2017. 


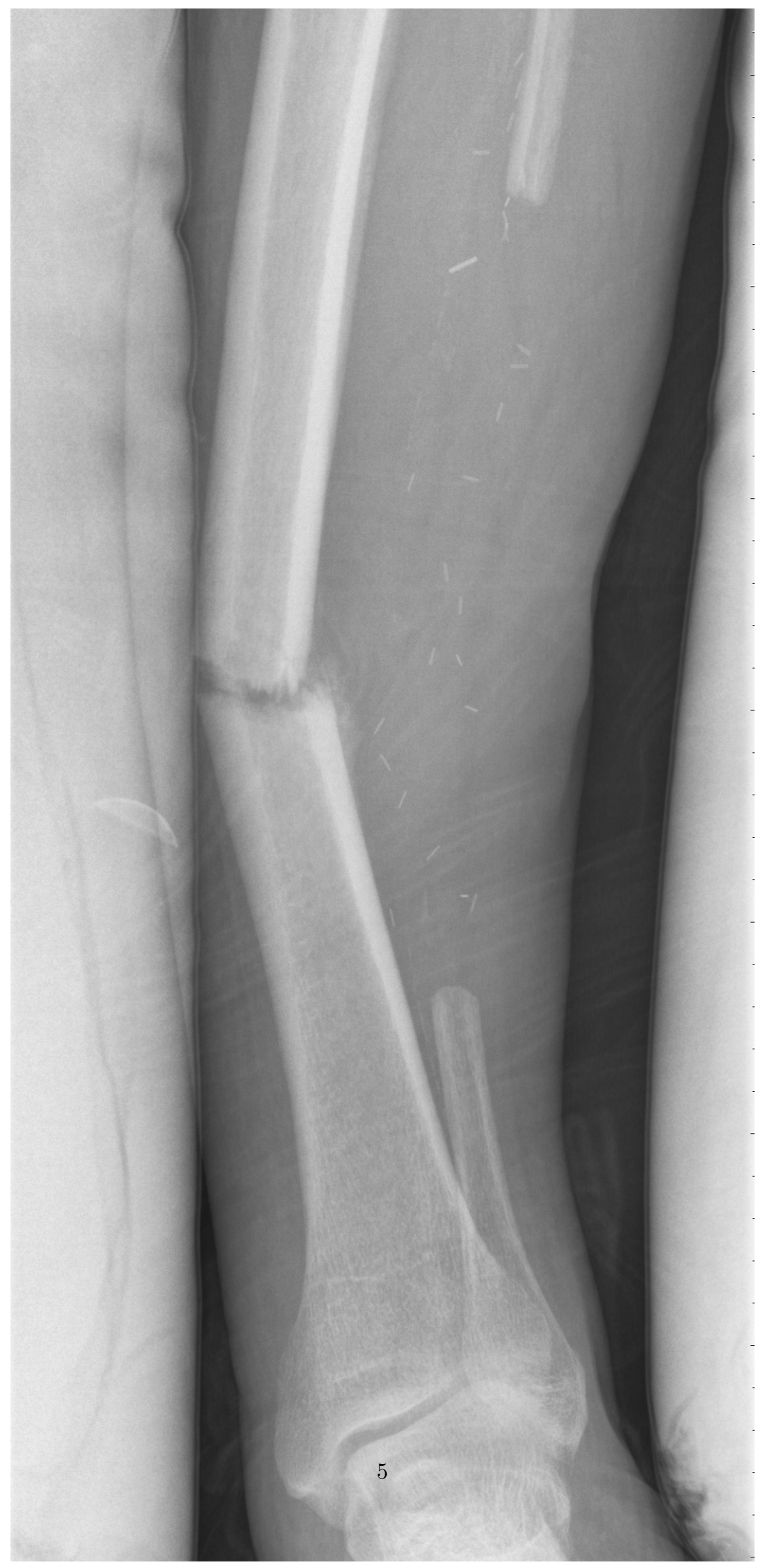




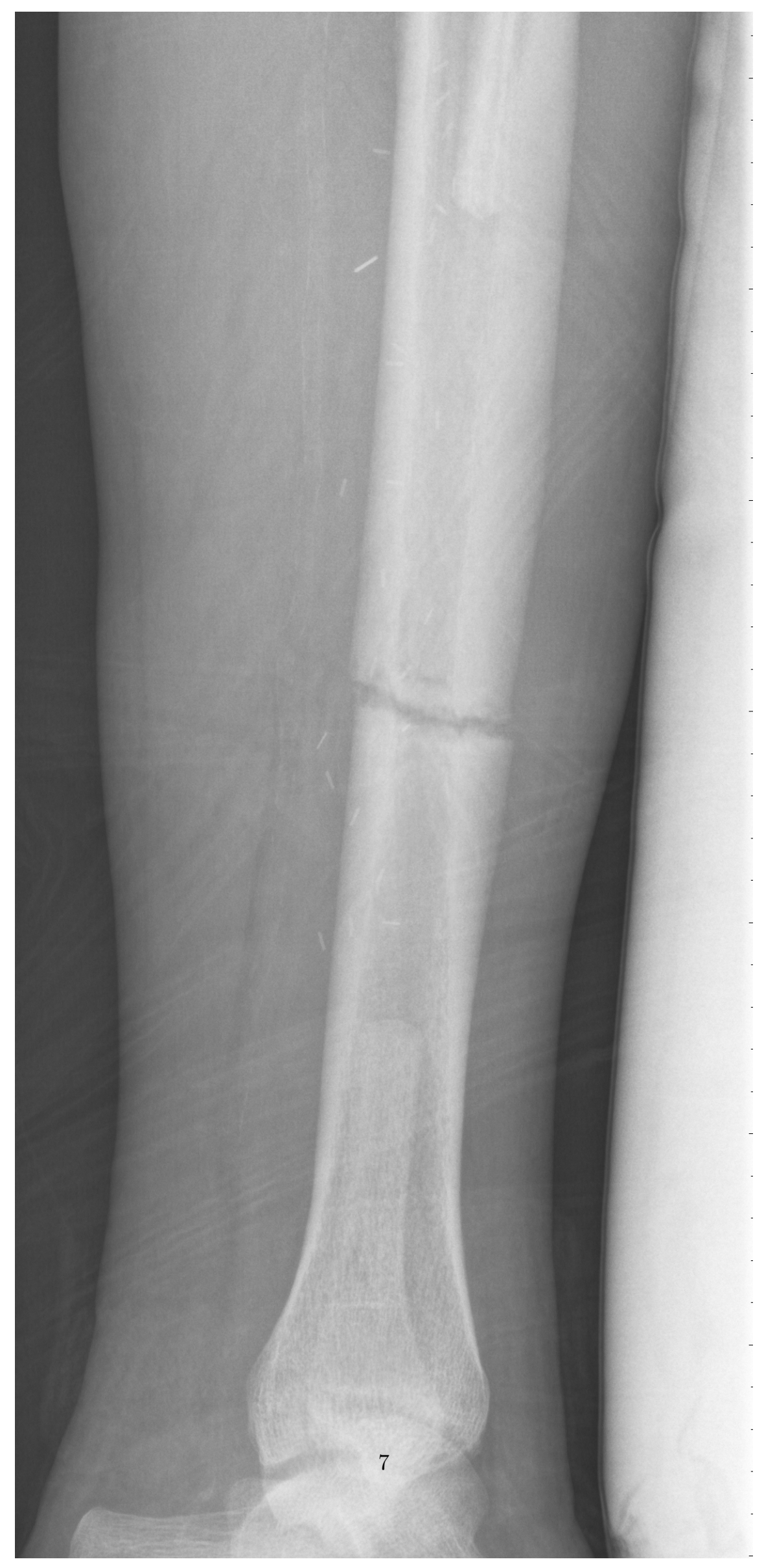




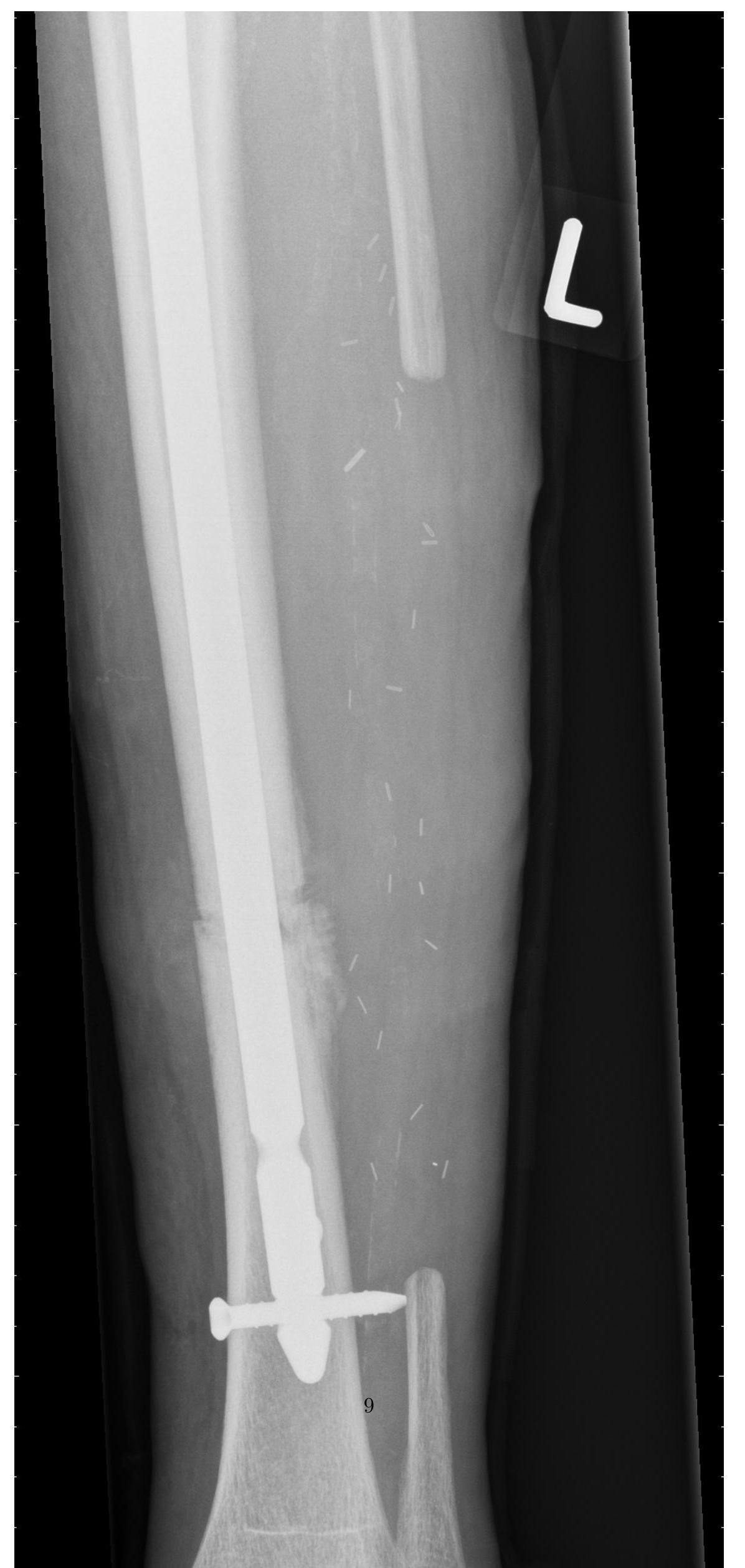




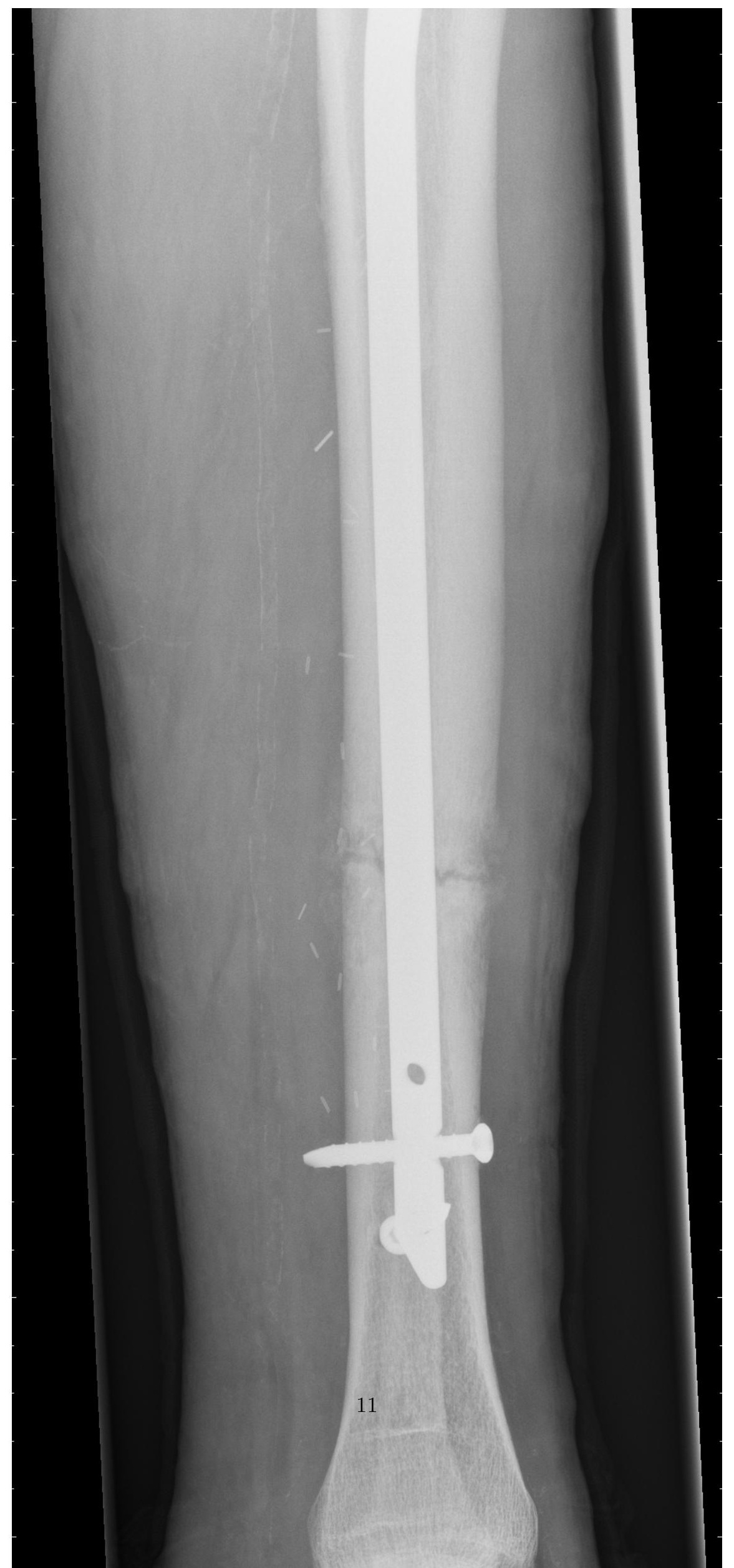




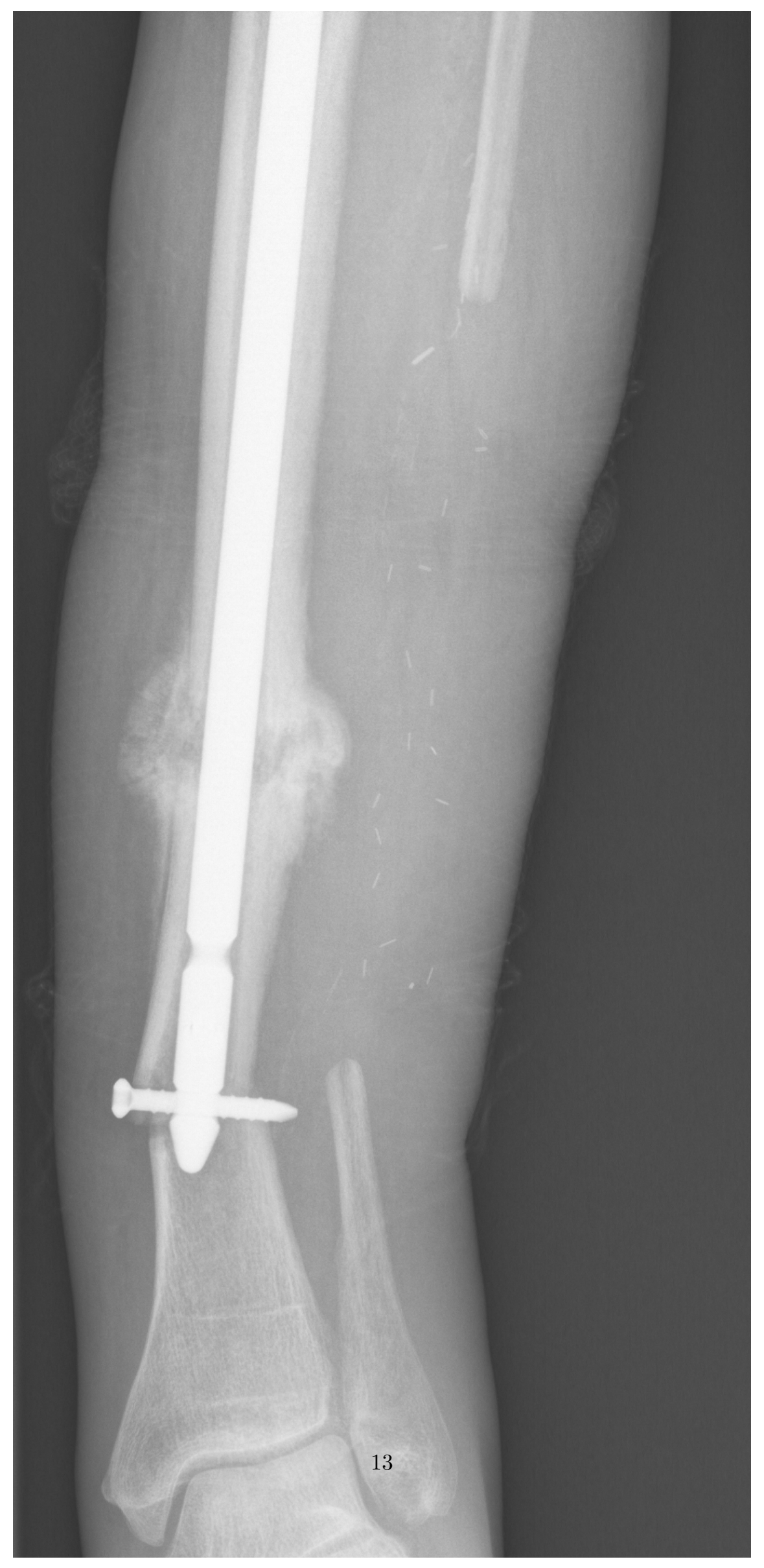




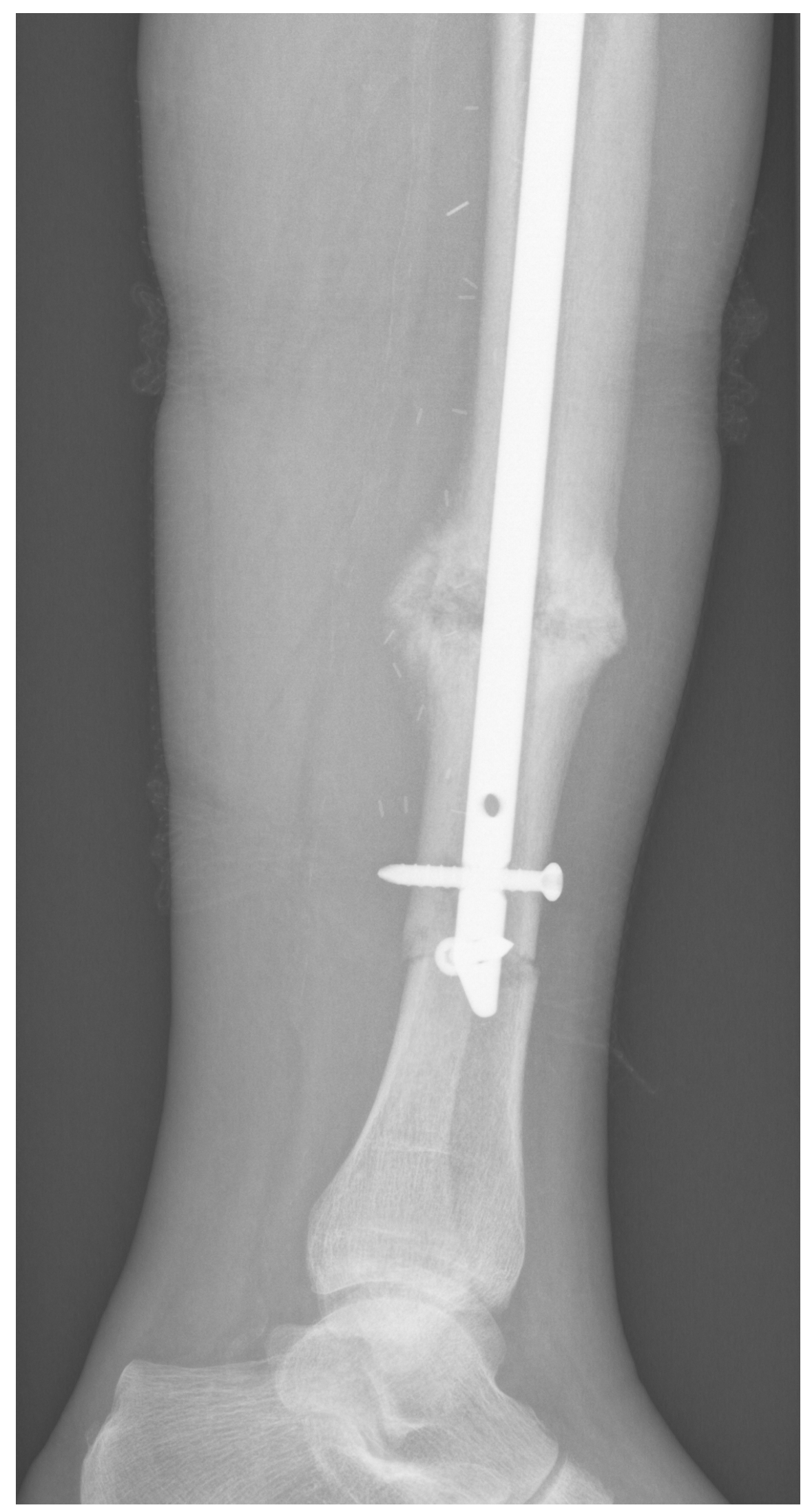





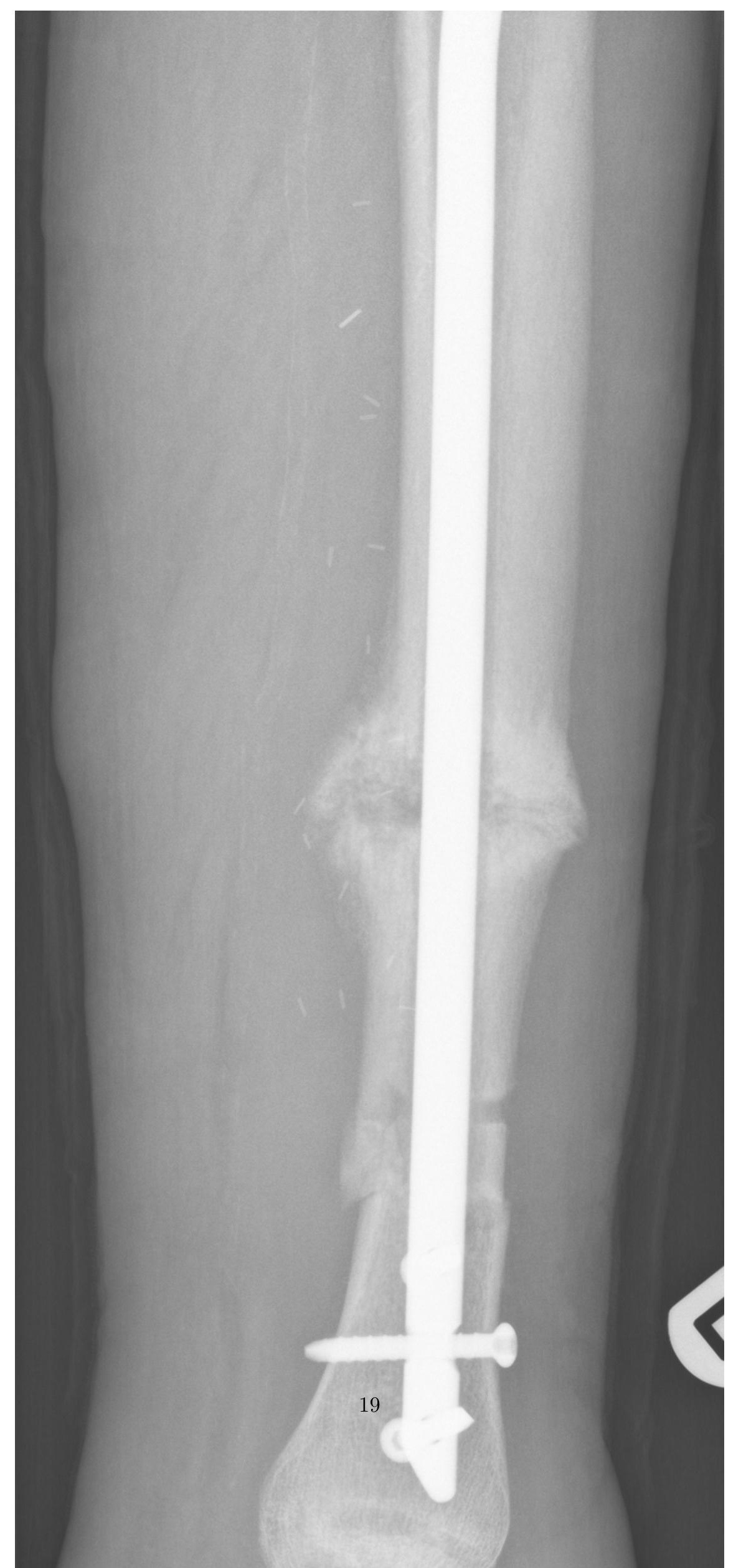





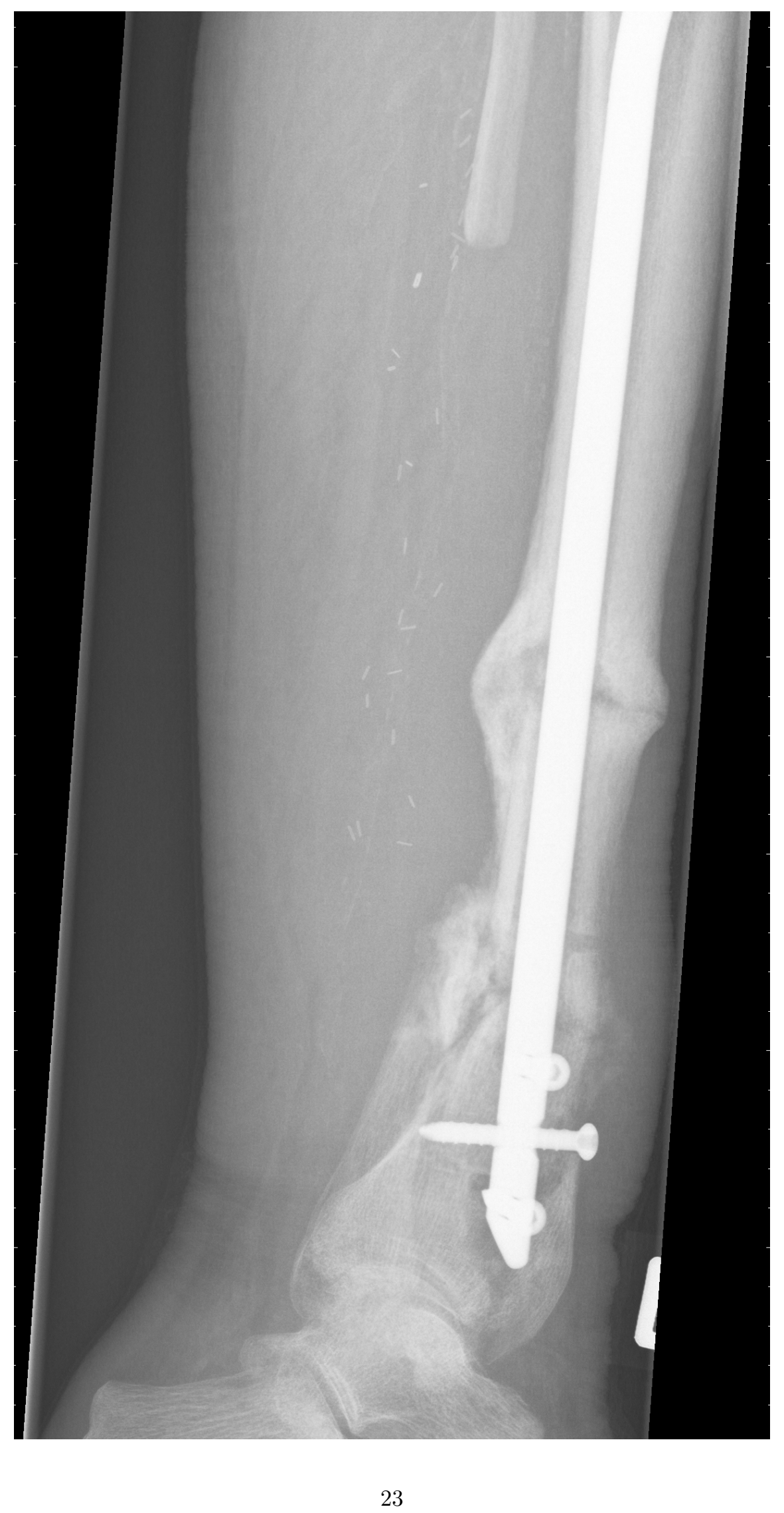




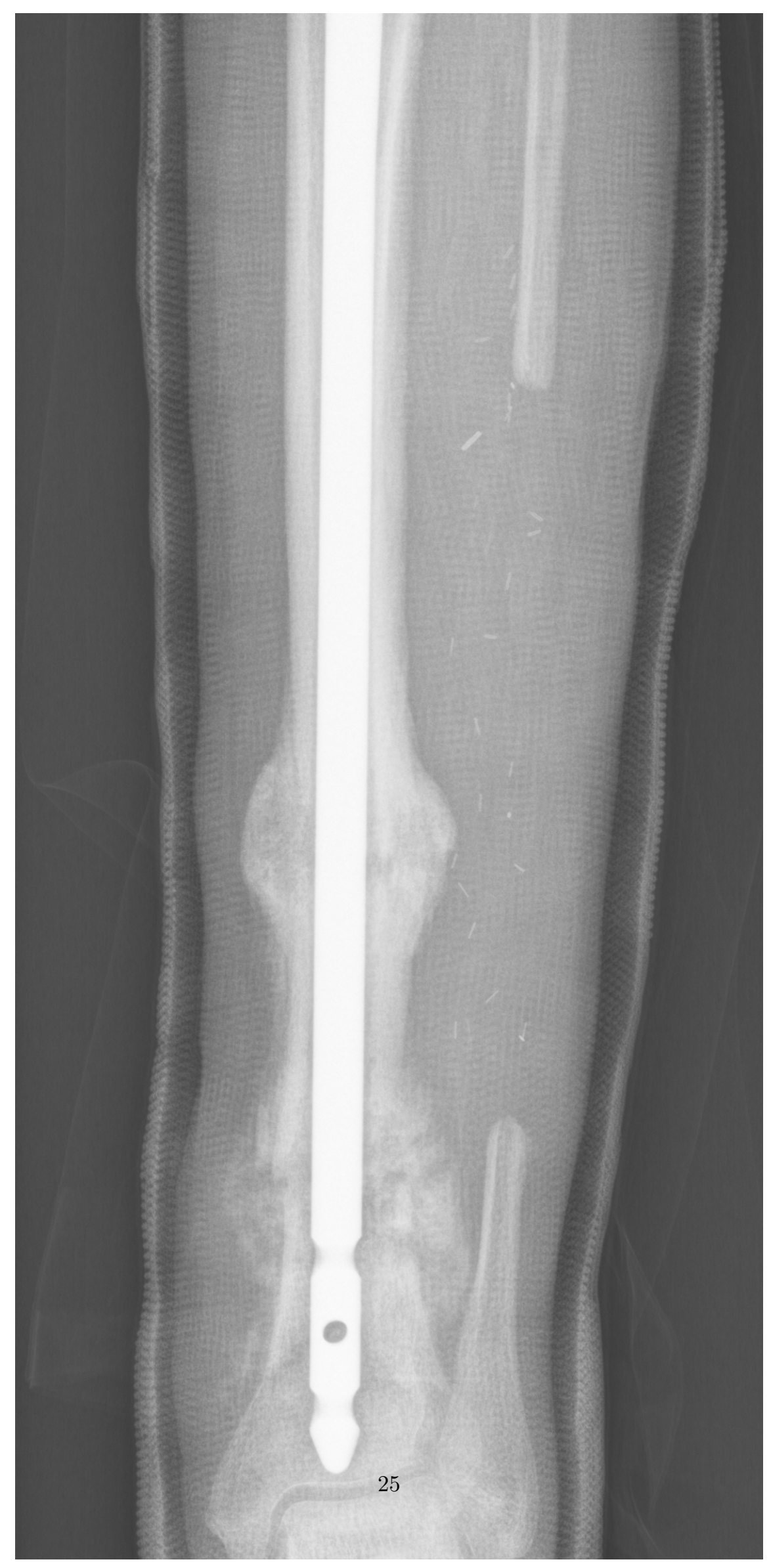




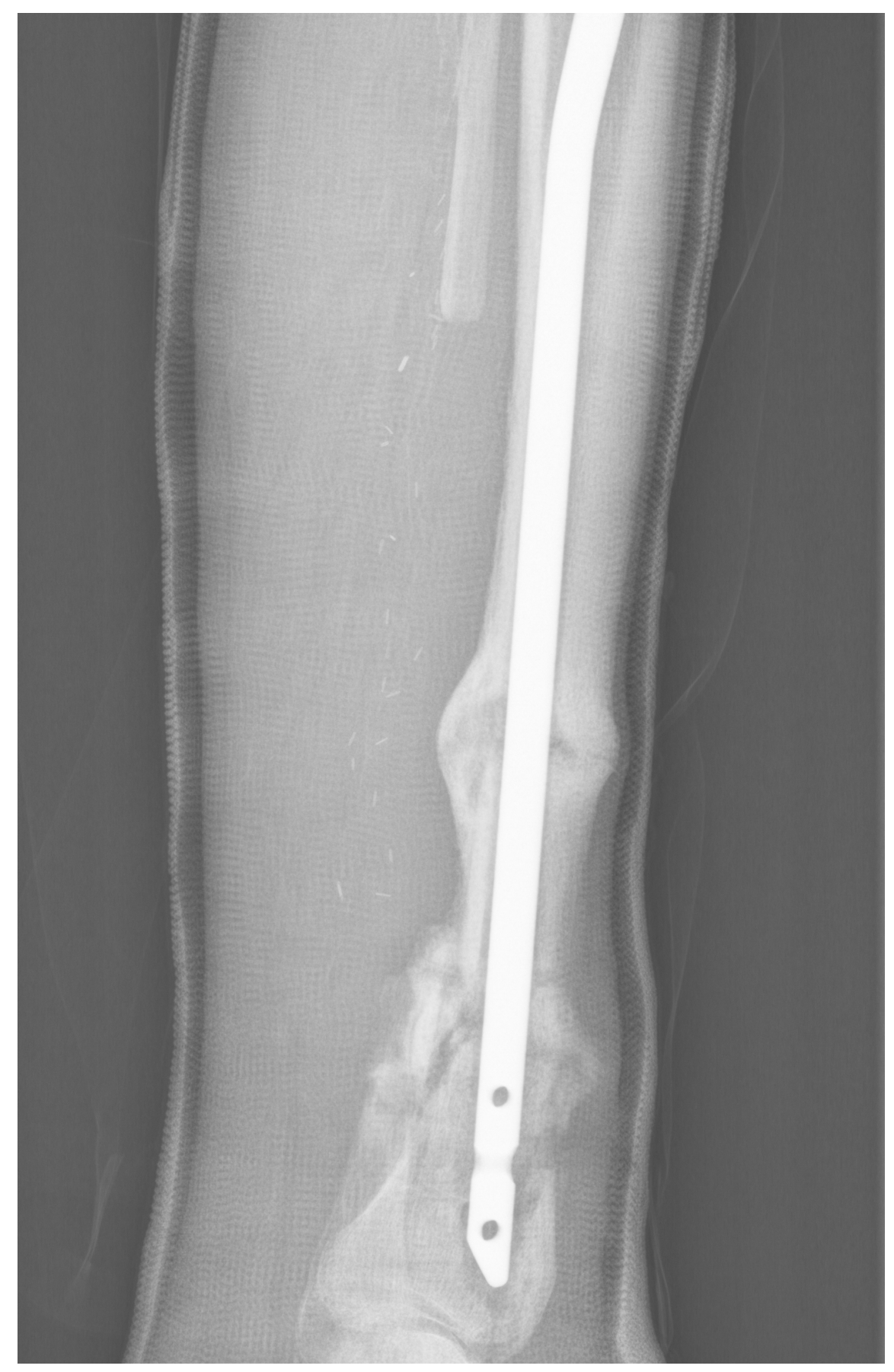



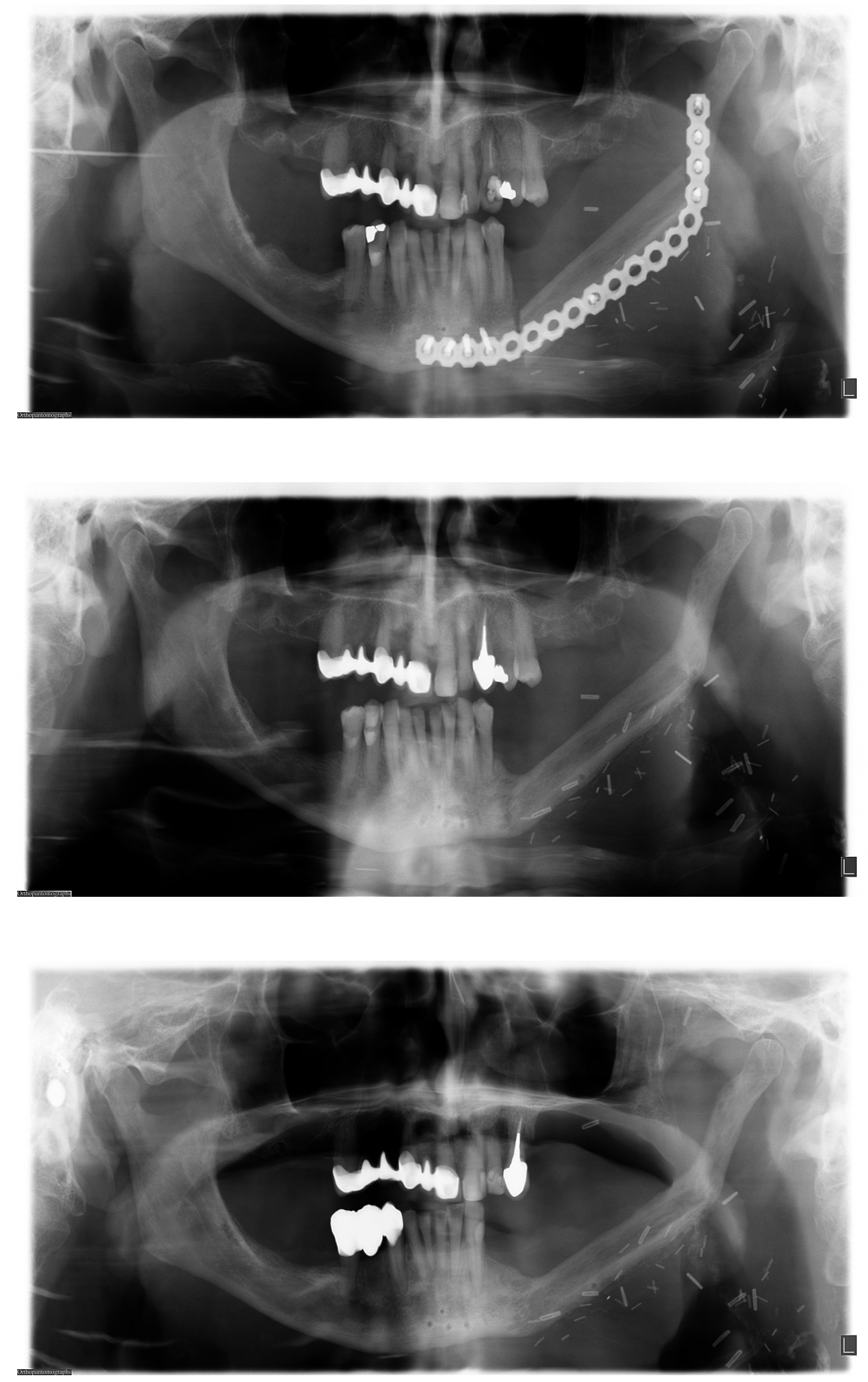